\title{
Effect of Epiretinal Membrane Peeling on Intravitreal Aflibercept Therapy Response for Polypoidal Choroidal Vasculopathy: A Case Report
}

\author{
Asli Kirmaci, Ali Demircan, Dilek Yasa, Zeynep Alkin \\ University of Health Sciences, Beyoglu Eye Training and Research Hospital, Istanbul, Turkey
}

\begin{abstract}
Traction forces in cases of epiretinal membrane (ERM) may antagonize the effects of anti-vascular endothelial growth factors (anti-VEGF) treatments and cause pharmacological resistance in patients with neovascular age-related macular degeneration. Presently described is a case with polypoidal choroidal vasculopathy (PCV) in conjunction with ERM who demonstrated a partial response to intravitreal aflibercept injections. A pars plana vitrectomy with ERM peeling was performed. After surgery, optical coherence tomography showed an improvement in the macular morphology and complete resolution of subretinal fluid. His visual acuity remained stable. Vitrectomy may improve the anti-VEGF response in some patients with ERM who do not respond to anti-VEGF therapy for PCV.

Keywords: Aflibercept, epiretinal membrane, polypoidal choroidal vasculopathy.
\end{abstract}

\section{Introduction}

Polypoidal choroidal vasculopathy (PCV) is a variant of neovascular age-related macular degeneration (nAMD) characterized by exudative and hemorrhagic changes within the macula leading to visual impairment with a prevalence that increases with age. This subtype of AMD is less responsive to treatment with anti-vascular endothelial growth factors (anti-VEGF) (I). In these refractory cases, aflibercept theraPy, a relatively new agent among anti-VEGF treatment alternatives, may be an option (2).

Idiopathic epiretinal membrane (ERM) is another cause of visual acuity deterioration due to traction forces on the retinal surface in the same group of older patients. The coexistence of the aforementioned conditions may cause difficulties in the management of PCV with anti-VEGF therapy (3).

In this case of a patient with both PCV and ERM, there was a response to intravitreal aflibercept injections before and after a pars plana vitrectomy with ERM peeling. To the best of our knowledge, this is the first report demonstrating the results of ERM peeling in a patient with PCV.

\section{Case Report}

An otherwise healthy, 68-year-old patient presented with reduced central vision in his left eye in November 2007. Patient's medical and ocular history was unremarkable. At the time of presentation, his best corrected visual acuity (BCVA) was 20/30 in the right eye and 20/66 in the left eye. Intraocular pressure was $13 \mathrm{mmHg}$ in the right eye, and $14 \mathrm{mmHg}$ in the left eye according to Goldmann applanation tonometry measurements. An anterior segment examination of both eyes was normal. A fundus examination revealed hard and soft drusen in the macula in both eyes (Fig. Ia). Fluorescein angiography demonstrated multiple hyperfluorescent spots in the macula due to the presence of drusen in the 

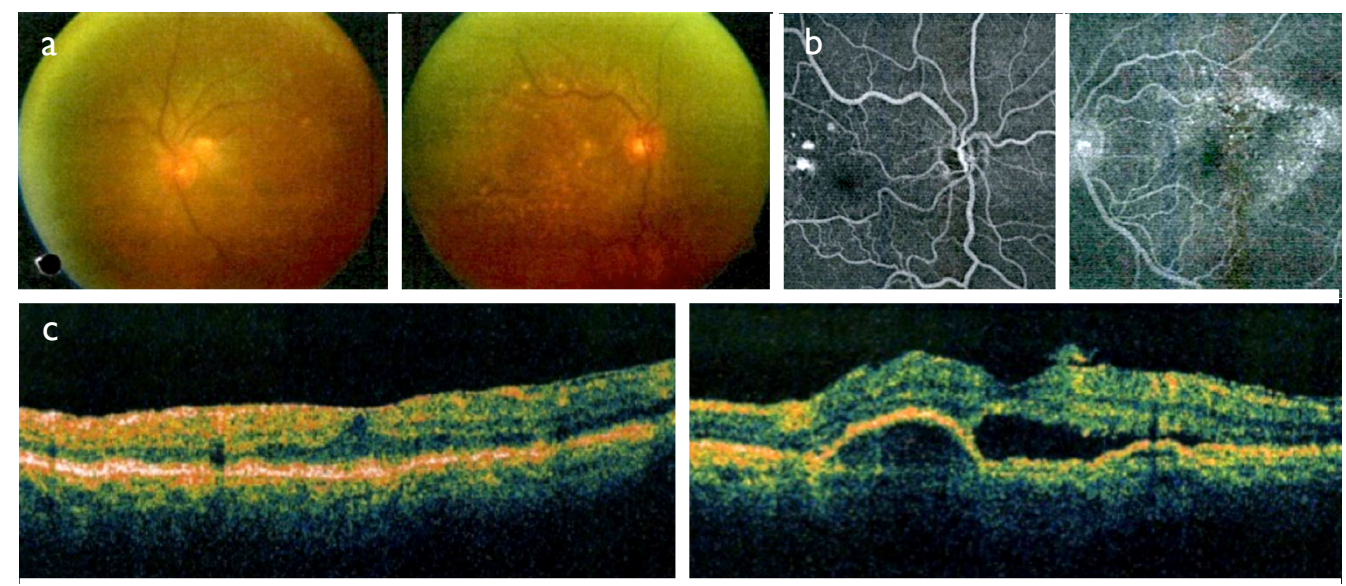

Figure I. (a) Fundus examination of the right and left eye revealing hard and soft drusen. (b) Fluorescein angiography shows multiple hyperfluorescent spots in the macula in the right eye and hyperfluorescence and leakage due to occult choroidal neovascular membrane in the left eye. (c) Optical coherence tomography indicating a thin epiretinal membrane in the right eye and pigment epithelial detachment with subretinal fluid in the left eye.
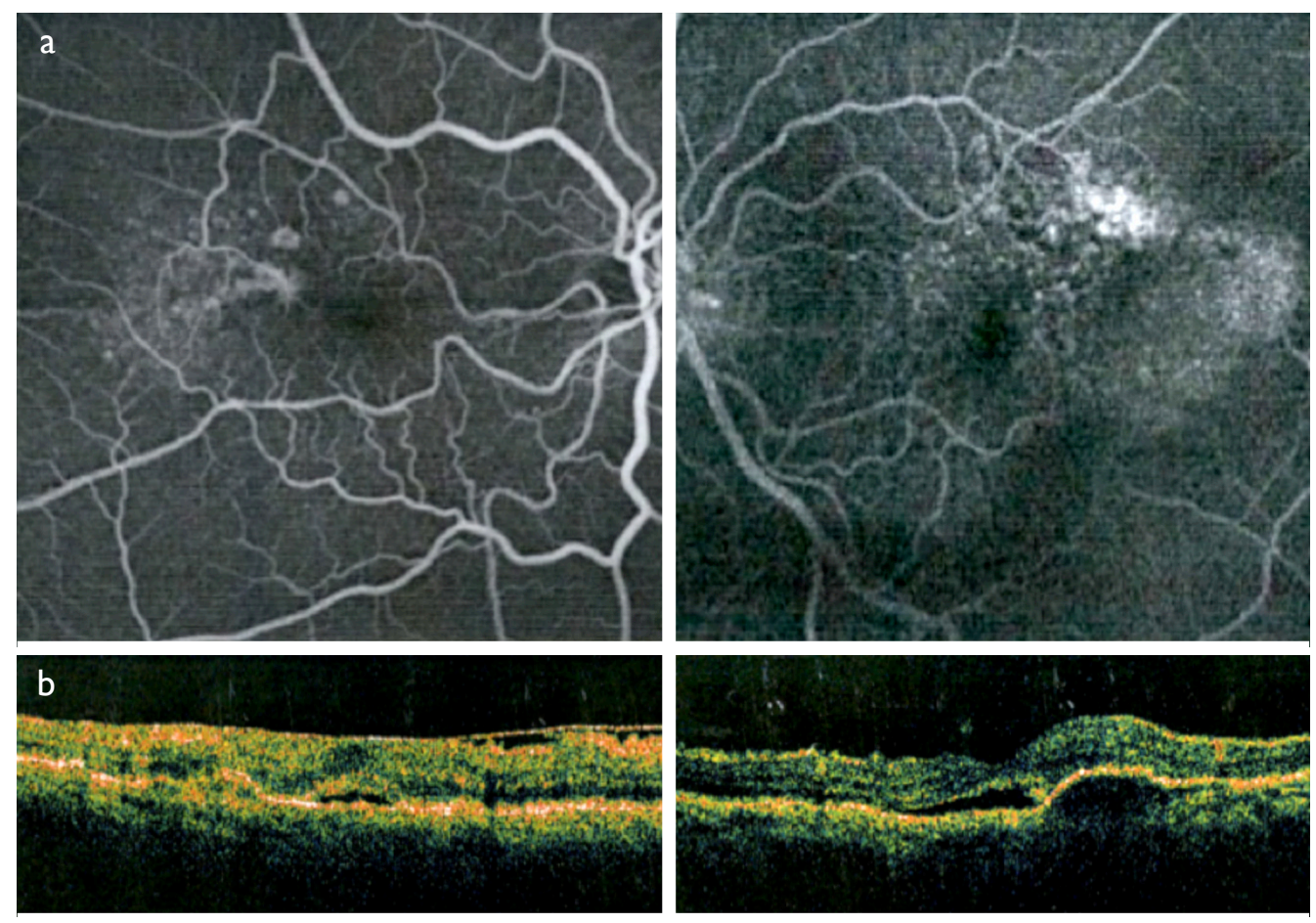

Figure 2. (a) Fluorescein angiography demonstrating hyperfluorescence and leakage related to the occult choroidal neovascular membrane in both eyes. (b) Optical coherence tomography illustrating the epiretinal membrane and subretinal fluid in the right eye.

right eye and hyperfluorescence and leakage resulting from occult choroidal neovascular membrane in the left eye (Fig. Ib). Optical coherence tomography (OCT) (Spectralis; Heidelberg Engineering, Heidelberg, Germany) demonstrated a thin ERM in the right eye, and pigment epithelial detachment (PED) and subretinal fluid in the left eye (Fig. Ic). He was diagnosed with dry AMD and ERM in the right eye and neovascular AMD in the left. He was treated with intravitreal bevacizumab injections in the left eye. In July 2009, his BCVA decreased to $20 / 50$ in the right eye. Fluorescein angiography revealed hyperfluorescence and leakage related to occult choroidal neovascularization in both eyes (Fig. 2a). OCT imaging demonstrated ERM and subretinal fluid in the right eye (Fig. 2b). He was advised to have 3 consecutive intravitreal bevacizumab injections for neovascular AMD in his right eye. Between July 2009 and August 2015, he underwent 18 bev- 

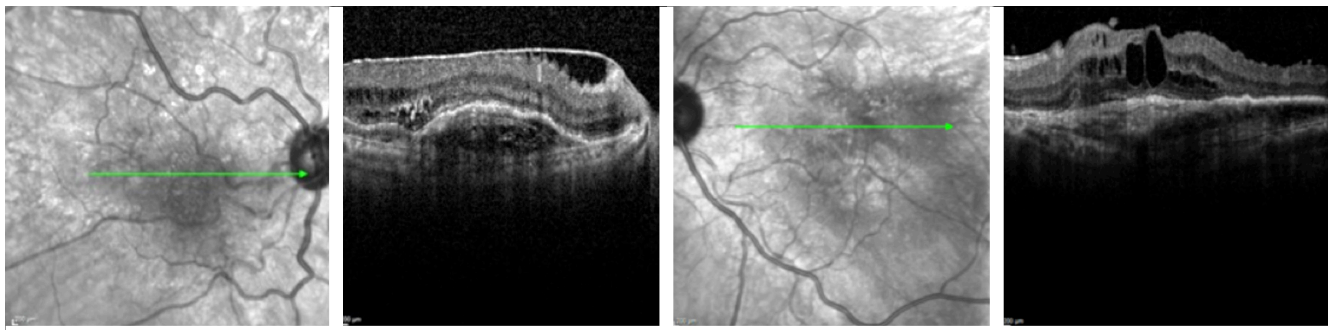

Figure 3. Optical coherence tomography shows the persistence of subretinal fluid in the right eye and intraretinal fluid in the left eye after bevacizumab injections.
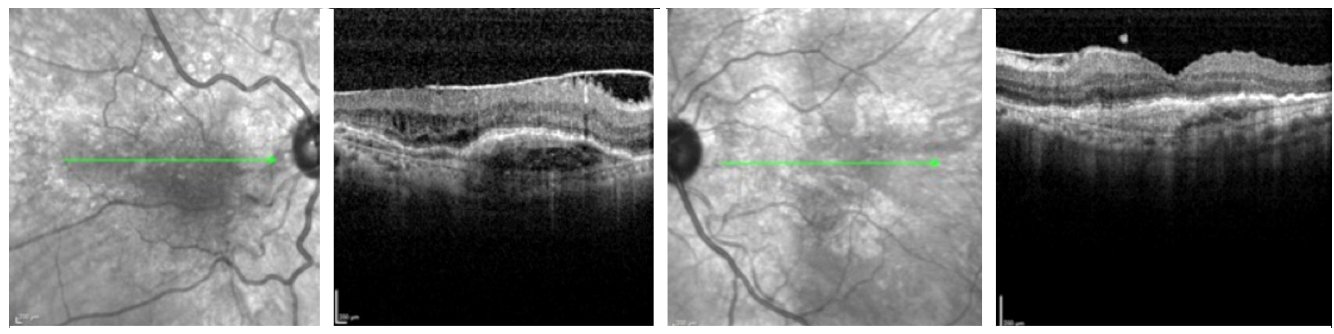

Figure 4. Optical coherence tomography demonstrates partial improvement in the subretinal fluid in the right eye and total resolution of the intraretinal fluid in the left eye.
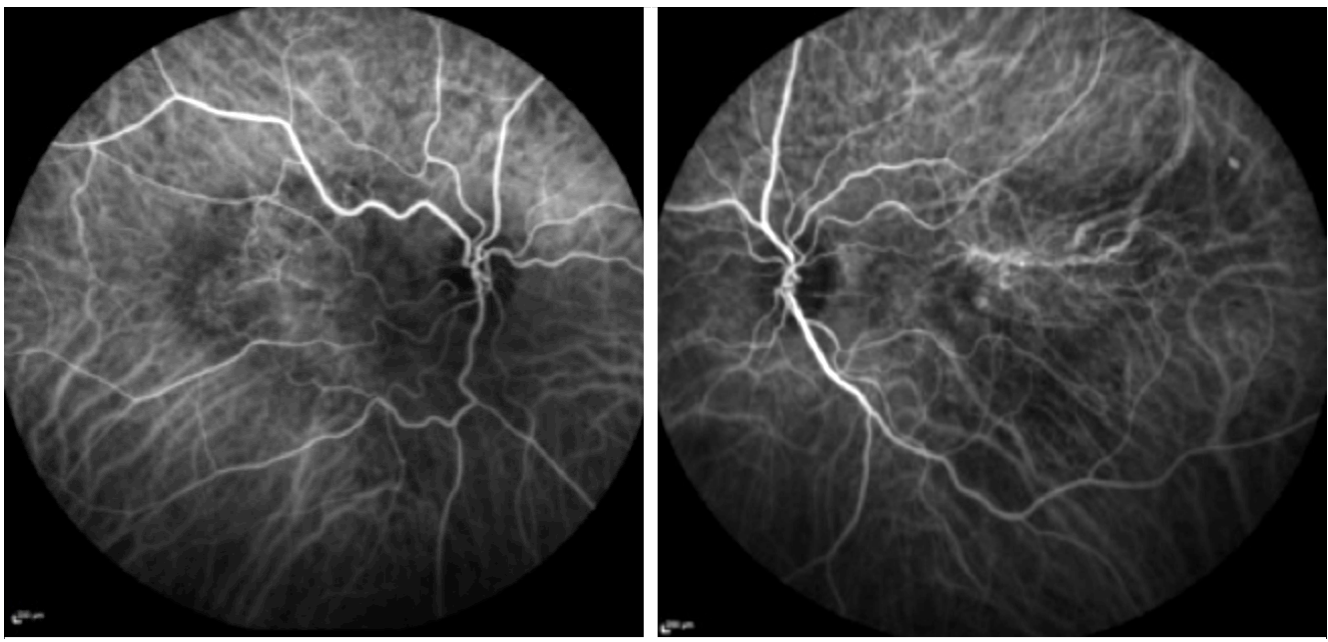

Figure 5. Indocyanine green angiography reveals a large branching vascular network with multiple polyps in both eyes.

acizumab injections for the right eye and 19 bevacizumab injections for the left eye. His BCVA remained stable at 20/66 in the right eye and $20 / 50$ in the left eye during this period. Since subretinal fluid in the right eye and intraretinal fluid in the left eye persisted despite the large number of bevacizumab injections, a decision was made to switch to intravitreal aflibercept therapy (Fig. 3). Between August 2015 and June 2016, the patient underwent 3 intravitreal aflibercept injections in each eye. His BCVA dropped to 20/100 in the right eye, while it was stable in the left. OCT imaging demonstrated that the intraretinal fluid totally resolved in the left eye, while the right eye showed partial improvement in subretinal fluid (Fig. 4). Indocyanine green angiography revealed a large branching vascular network with multiple polyps in both eyes (Fig. 5). The presence of ERM was thought to prevent treatment response in the right eye. Phacoemulsification and intraocular lens implantation together with pars plana vitrectomy and ERM peeling was performed in the right eye. After the surgery, I more intravitreal aflibercept injection was administered in the right eye, and 2 additional aflibercept injections were administered in the left eye. The final BCVA was $20 / 100$ in the right eye and 20/50 in the left eye. OCT images illustrated complete resolution of the intraretinal fluid in the right eye at the last visit (Fig. 6).

\section{Conclusion}

We observed an improvement in macular morphology after the removal of an ERM in a patient with PCV who showed 

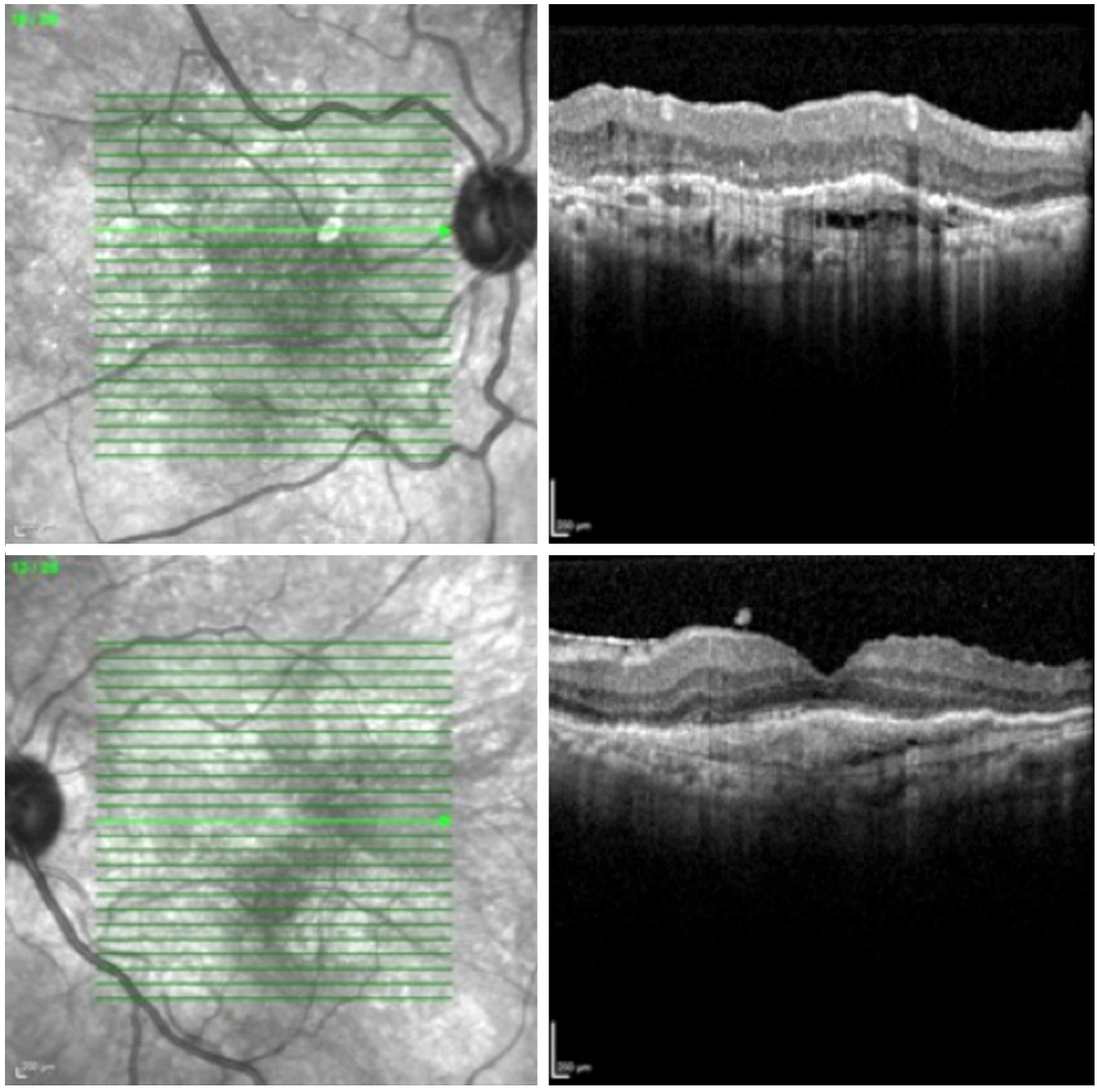

Figure 6. Optical coherence tomography shows complete resolution of the intraretinal fluid in the right eye at the last visit.

a partial response to intravitreal aflibercept injections. OCT imaging revealed a complete resolution of subretinal fluid after the surgery, though there was no progress in the functional results, as the BCVA remained stable following the surgery. Previous studies have demonstrated that the presence of an ERM leads to an increase in the number of anti-VEGF injections and a decrease in the injection interval in the management of nAMD (4).

There are several factors regarding idiopathic ERM that may have an effect upon both the progression and the treatment response in the management of PCV. First of all, ERM formation contributes to the already existing chronic inflammation in eyes with PCV. Mechanical traction may cause a deterioration of the retina pigment epithelium (RPE) or Bruch's membrane and induce a release of VEGF and other mediators. While the traction forces created by ERM stimulate the progression of nAMD, several studies have demonstrated that patients with AMD have a greater rate of vitreomacular interface abnormalities, including ERM, compared with those without AMD (3). Pierro et al. (5) indicated in their study that ERM was present in $26 \%$ to $32 \%$ of patients with nAMD. The inflammation present in nAMD likely leads to the formation of ERM, and this increased inflammation induces the progression of nAMD (4). Secondly, apart from the inflammatory cytokine-induced pathway, the VEGF concentration in the aqueous humor is also increased by hypoxia due to the closure of small vessels around the macular area as a result of the traction (6). Therefore, a vitrectomy with an ERM peel can be a beneficial procedure for PCV patients with ERM in that it may reduce inflammatory mediators and provide greater oxygen tension in the vitreous cavity $(7,8)$. We also performed a pars plana vitrectomy procedure with ERM removal in our case, and observed reduced progression of the disease after the surgery.

The presence of an ERM and the associated traction is thought to be influential in the treatment responsiveness of nAMD patients. Traction forces may lead to a breakdown in the architecture of the RPE, Bruch's membrane, and photore- 
ceptor layer, which may result in a reduction in the effectiveness of anti-VEGF agents (4). In addition, the presence of a membrane may act as a physical barrier and result in reduced penetration of the anti-VEGF agents through these membranes, leading to pharmacological resistance to anti-VEGF treatment $(3,4)$. In our case, we continued treatment with intravitreal aflibercept injections after the surgery and observed an improvement in anatomical results, although the BCVA remained the same as before the surgery.

In summary, we speculate that pars plana vitrectomy with ERM peeling seems to have been beneficial in the management of a PCV patient with ERM by both reducing the progression of the disease and increasing the response to treatment. Along with the conventional parameters, such as the presence of intraretinal fluid, hemorrhage, and pigment epithelial detachment, the presence of ERM might be considered at baseline in the treatment of a PCV patient.

\section{Disclosures}

Informed consent: Written informed consent was obtained from the patient for the publication of the case report and the accompanying images.

Peer-review: Externally peer-reviewed.

Conflict of Interest: None declared.

Authorship Contributions: Involved in design and conduct of the study (ZTA, AK); preparation and review of the study (AK); data collection (AD, DY).

\section{References}

I. Kokame GT, Lai JC, Wee R, Yanagihara R, Shantha JG, Ayabe J, et al. Prospective clinical trial of Intravitreal aflibercept treatment for Polypoldal choroidal vasculopathy with hemorrhage or exudation (EPIC study): 6 month results. BMC Ophthalmol 2016;16:127. [CrossRef]

2. Kawashima Y, Oishi A, Tsujikawa A, Yamashiro K, Miyake M, Ueda-Arakawa $\mathrm{N}$, et al. Effects of aflibercept for ranibizumab-resistant neovascular age-related macular degeneration and polypoidal choroidal vasculopathy. Graefes Arch Clin Exp Ophthalmol 20I5;253:|47|-7. [CrossRef]

3. Alkin Z, Ozkaya A, Ayranci Osmanbasoglu O, Agca A, Karakucuk Y, Yazici AT, et al. The Role of Epiretinal Membrane on Treatment of Neovascular Age-Related Macular Degeneration with Intravitreal Bevacizumab. ScientificWorldjournal 2013;2013:958724. [CrossRef]

4. Karaca EE, Kepez Yldz B, Çubuk MÖ, Özdek Ş. Epiretinal Mebranes in Neovasculer Age-Related Macular Degeneration: Effect on Outcomes of Anti-vascular Endothelial Growth Factor Therapy. Retina 2015;35:1540-6. [CrossRef]

5. Pierro L, Zampedri E, Milani P, Gagliardi M, Isola V, Pece A. Spectral domain OCT versus time domain OCT in the evaluation of macular features related to wet age-related macular degeneration. Clin Ophthalmol 2012;6:219-23.

6. Schulze S, Hoerle S, Mennel S, Kroll P. Vitreomacular traction and exudative age-related macular degeneration. Acta Ophthalmol 2008;86:470-8I. [CrossRef]

7. Roller AB, Mahajan VB, Boldt HC, Abramoff MD, Russell SR, Folk JC. Effects of vitrectomy on age-related macular degeneration. Ophthalmology 2010;1 17:1381-6. [CrossRef]

8. Kimura S, Morizane Y, Toshima S, Hosogi M, Kumase F, Hosokawa $M$, et al. Efficacy of vitrectomy and inner limiting membrane peeling in age-related macular degeneration resistant to anti-vascular endothelial growth factor therapy, with vitreomacular traction or epiretinal membrane. Graefes Arch Clin Exp Ophthalmol 2016;254:173I-6. [CrossRef] 